\title{
Theory reduction by means of functional sub-types
}

\author{
Michael Esfeld \& Christian Sachse \\ University of Lausanne, Department of Philosophy \\ CH-1015 Lausanne, Switzerland \\ Michael-Andreas.Esfeld@unil.ch, Christian.Sachse@unil.ch \\ published in International Studies in the Philosophy of Science 21 (2007), pp. 1-17
}

\section{Abstract}

The paper sets out a new strategy for theory reduction by means of functional sub-types. This strategy is intended to get around the multiple realization objection. We use Kim's argument for token identity (ontological reductionism) based on the causal exclusion problem as starting point. We then extend ontological reductionism to epistemological reductionism (theory reduction). We show how one can distinguish within any functional type between functional sub-types. Each of these sub-types is coextensive with one type of realizer. By this means, a conservative theory reduction is in principle possible despite multiple realization. We link this account with Nagelian reduction, as well as Kim's functional reduction.

\section{Ontological and epistemological reductionism}

Since the early 1970s, the received view has been that the argument from the multiple realization of the properties in which the special sciences trade refutes reductionism. Functionalism, conceived as non-reductive physicalism, used to be the standard position in the metaphysics of science, including the metaphysics of mind. In recent years, that consensus has been challenged, in particular consequent upon a renewed focus on the problem how the properties that the special sciences consider can be causally efficacious. Due to the work of Jaegwon Kim among others (notably Kim 1998, 2005), the issue of reductive vs. nonreductive physicalism is again open.

The aim of this paper is to sketch out a new general strategy for reduction despite multiple realization. By reductionism, we understand two theses:

- Ontological thesis: All there is in the world are entities that are identical with fundamental physical tokens and their configurations. Some of these configurations are tokens of properties in which the special sciences trade, that is, chemical, geological, biological, neurobiological, psychological, etc., tokens.

- Epistemological thesis: It is in principle possible to reduce every true description of what there is in the world to a description constructed in terms of fundamental physics.

We conceive reductionism as a conservative position: we recognize the property tokens of the special sciences as existing in the same way as do the fundamental physical property tokens, and we acknowledge that the entities in the world make the theories of the special sciences true in the same way as the fundamental physical theories. The point of epistemological reductionism is to show how all these theories fit together in a coherent system of our knowledge, not to eliminate any one of them. Our argument is based on three premises.

(1) Ontological reductionism is well motivated by the causal exclusion problem. The property tokens of the special sciences are causally efficacious. However, there is a problem generated by the following three principles: (i) The property tokens of the special sciences strongly supervene on the fundamental physical property tokens. (ii) Any physical token has a sufficient physical cause insofar as it has a cause at all. (iii) There is no systematic overdetermination. Consequently, the physical tokens exclude the property tokens of the 
special sciences from being causally efficacious - unless one maintains that these are identical with configurations of physical tokens. If the property tokens of the special sciences are identical with configurations of physical tokens and if causes are property tokens, it does not make sense to wonder whether the cause brings about its effect qua being physical or qua being, say, biological. The cause is biological qua being physical, namely qua being a certain configuration of - in the last resort - fundamental physical tokens. Any token of a property in which the special sciences trade can be identical only with a configuration of fundamental physical tokens, because any property token of the special sciences stretches over a spacetime region. Fundamental physical properties, by contrast, can be instantiated at space-time points. Token identity as solution to the causal exclusion problem has been argued for by Kim (1998, 2005). Kim's argument is hotly disputed, notably in the philosophy of mind. We do not have the space to go into that dispute here. We simply accept ontological reductionism (token identity) as a starting point for this paper.

(2) All there exists in the world are property tokens. Property types are concepts that we use in order to seize salient similarities among property tokens. If one were to admit anything over and above property tokens - such as, notably, property types (universals) existing in the world -, one would fall back into the causal exclusion problem as regards the property types. There are of course other important arguments for recognizing only tokens as existing in the world and taking types to be concepts. Again, we do not have the space here to go into these arguments. We simply state our sympathy with, for instance, John Heil's deflationary ontology (Heil 2003).

(3) Ontological and epistemological reductionism entail one another. Epistemological reductionism implies ontological reductionism: if it is in principle possible to reduce every true description of what there is in the world to a description in terms of fundamental physics, then all there is in the world are entities that are identical with fundamental physical tokens and their configurations. Ontological simplification is one main goal of theory reduction. Ontological reductionism, for its part, implies that one and the same entities (property tokens) make true physical descriptions as well as descriptions in terms of the special sciences. If there were something in the latter descriptions that can in principle not be reduced to physics, then there would be something about the entities that make true these descriptions that would not be identical with these entities being configurations of physical property tokens. Consequently, ontological reductionism would be false. Hence, ontological and epistemological reductionism stand or fall together.

The task we set ourselves in this paper is to make a case for epistemological reductionism given ontological reductionism. We shall consider only the ideal situation of true theories of the special sciences being reduced to a true and universal physical theory. Of course, the reduction to a fundamental and universal theory of physics is only the ultimate goal. There is no point in, for instance, trying to reduce psychology directly to physics. The terms "theory of the special sciences" and "universal physical theory" are only intended to indicate a general scheme for which terms such as "psychology" and "neurobiology" or "genetics" and "molecular biology", etc., can be put in as well.

Furthermore, in real cases of a theory reduction, the reduction usually amounts to a correction of the reduced theory. Consequently, what is in fact reduced is a corrected version of the theory targeted for reduction. However, our aim is a general philosophical one instead of examining concrete examples of theory reduction: we side with the friends of the special 
sciences in accepting the theories of the special sciences as true and good theories, containing law-like generalizations, explanations, etc., that do not call for a correction from physics. We set out to show that even on this basis, these theories are in principle reducible to physics and in that way integrated into a coherent system of our knowledge. Thus, our aim is to rebut philosophical objections against the principled possibility of intertheoretic reduction, notably the objection from multiple realization. We intend to establish reductionism as a stable philosophical position that stirs a middle course between anti-reductionism and eliminativism.

In the next section, we briefly present the state of the art by discussing the idea of functional reduction. We then set out our proposal for epistemological reduction by means of functional sub-types (section 3) and indicate a biological illustration of that proposal (section 4). Finally, we assess that proposal as regards intertheoretic reduction in general (section 5).

\section{2. $\quad$ Functional reduction}

How is it possible that one and the same entity makes true a fundamental physical description as well as descriptions in terms of one or more of the special sciences? A promising proposal to answer this question is functional reduction. This proposal considers the properties in which the special sciences trade to be functional properties. We shall limit ourselves to functional properties in this paper. Drawing on David Lewis (1970), Kim develops an account of functional reduction (2005, chapter 4, notably 101-102; see also Chalmers 1996, 42-51). This account can be reconstructed by means of the following three steps:

1) Any functional property $F$ is defined in terms of a causal role. That definition is couched in the vocabulary of one of the special sciences. The causal role requires a realization by some configuration or other of in the last resort fundamental physical tokens.

2) One discovers configurations of physical tokens that realize $F$, because they bring about the effects that characterize $F$. The description of these configurations is couched in a physical vocabulary, not the functional one. As has recently been pointed out (see Endicott 1998, section 8; Hüttemann 2003, chapter 4.3; Marras 2005, 344-347), functional reduction is therefore committed to bridge principles: these are one-way conditionals that map the physical description of configurations of tokens that realize $F$ to the description of tokens of $F$ in the functional vocabulary.

3) Given step 2, one shows how the physical description of any such configuration of tokens explains why the configuration in question is a case of $F$. This is a causal explanation, bringing out how the configuration produces the effects that characterize $F$. If we accept the identity solution of the causal exclusion problem (Premise 1 in the last section), we have to conceive realization as token identity.

To illustrate that account, assume that genes are functional properties that are defined notably by their characteristic phenotypic effects. Considering a grossly oversimplified case just for the purpose of illustration, suppose that there is a gene whose characteristic phenotypic effect is to produce white blossoms at flowering time within plants of certain types (step 1). One then discovers that certain segments of DNA realize the causal role that defines genes for white blossoms (step 2). Molecular biology explains how the segments of DNA in question produce the phenotypic effect that defines the gene for white blossoms given appropriate conditions in the physical environment (step 3).

Functional reduction shows how for each single token, the functional description can in principle be reduced to in the last resort a fundamental physical description. Moreover, 
functional reduction establishes a systematic link between the different descriptions. Given a configuration of physical tokens that realizes the functional type $F$, any configuration of tokens that comes under the same physical type $P$ as that configuration is a case of $F$ as well. In other words, making true the physical description $P$ is a sufficient condition for making true the functional description $F$. By a realizer type $P$, we always understand a full realizer of $F$; consequently, it is not possible that something comes under the concept $P$ without coming under the concept $F$ (given certain normal conditions in the environment) (compare the distinction between "core realizers" and "total realizers" that Shoemaker 1981, section 2, draws). For instance, given a token segment of DNA that realizes the gene for white blossoms, any segment of DNA that comes under the same molecular biological description as that token segment is a realizer of the functional type "gene for white blossoms", too (taking for granted certain normal conditions in the physical environment). Thus, functional reduction contains bridge principles in the form of law-like generalizations that establish a link between physical realizer concepts and functional concepts. These are one-way conditionals of the form "If something comes under the physical type $P$, then it comes under the functional type $F$ " (whereby "physical" can be replaced with "chemical" or "molecular biological" or "neurobiological" in the appropriate context).

It is common ground that functional types admit of multiple realizations. The functional type "gene for white blossoms" can be realized by segments of DNA that come under different molecular types, because they differ in molecular composition. Consequently, we cannot turn the mentioned one-way conditionals into biconditionals. Coming under the physical type $P$ is a sufficient, but not a necessary condition for coming under the functional type $F$ (see notably Putnam 1967 / 1975; Fodor 1974; Hull 1974, chapter 1). As mentioned in Premise 2 in the last section, we treat property types as concepts. Realizing $F$ thus means coming under the concept $F$, and multiple realization is to say that entities that come under one and the same functional concept $F$ fall under different physical concepts $P_{1}, P_{2}, P_{3}$, etc.

Functional reduction as set out by Kim pays tribute to multiple realization. However, there remains a problem: the reduction of the functional description for each single token does not take the homogeneity of the functional tokens into account. Certain configurations that are composed in different manners of physical tokens make true one and the same functional description because they are similar in a salient way: they constitute a certain pattern that is the object of concepts and law-like generalizations of the special sciences, consisting notably in a homogeneous pattern of characteristic effects. Let us use the term "pattern" to capture the salient similarities among certain configurations of physical tokens in virtue of which they come under one functional concept.

As regards the functional patterns, Kim faces a dilemma: either the theories that focus on such patterns are irreducible, because there is more to these patterns than configurations of fundamental physical tokens, and then ontological reductionism is refuted. Or those patterns do not exist, and then reductionism is no longer conservative but eliminativist as regards the law-like generalizations and explanations of the special sciences. When pushed, Kim is prepared to endorse that eliminativist consequence (e.g. Kim 1999, 17-18; 2005, 26, 58), although he considers himself to be a conservative reductionist (Kim 2005, 159-160). To avoid this dilemma, we have to develop functional reduction into a fully-fledged theory reduction, going beyond the reduction of single descriptions. That is to say, we have to extend the mentioned one-way conditionals to biconditionals of the form "If and only if something 
comes under the physical type $P$, then it comes under the functional type $F$ ". But how can this be done given multiple realization?

\section{Reduction by means of functional sub-types: the argument}

In order to counter the argument that runs from multiple realization to an objection to epistemological reductionism, one has to establish a link between two manners of classification of configurations of fundamental physical tokens - classification by means of composition in physics and by means of functional role in many of the special sciences. The aim is to show that differences in composition go together with functional differences. However, this has to be done without infringing on the causal homogeneity of the functional patterns that are the object of law-like generalizations in the special sciences.

Our proposal takes up the criticism of the multiple realization argument put forward by William Bechtel, Jennifer Mundale and Robert McCauley. In brief, they maintain that (1) the idea of widespread multiple realization is an illusion arising from the fact that the functional description is coarse-grained, whereas the physical description of the realizer is more finegrained; if one uses the same degree of abstraction in the functional and the physical description, the impression of widespread multiple realization vanishes. (2) If there nonetheless are cases of multiple realization, we should try to introduce a more fine-grained functional classification (see Bechtel \& Mundale 1999, 201-204; McCauley 2007, section 8).

By contrast, as mentioned at the end of section 1, we accept for the purposes of this paper the special sciences as they stand and thus the standard form of multiple realization. Nonetheless, we intend to take up the latter suggestion (2) and to integrate it into a proposal that develops functional reduction into a fully-fledged and conservative theory reduction. Our argument is this one:

1) Consider configurations of physical tokens that come under one functional type (concept) $F$. Assume that there is a difference in composition among these configurations that amounts to these configurations coming under two different physical realizer types of $F$. In this case, there also is a systematic difference in the way in which these configurations bring about the effects that characterize $F$.

2) For any such difference there is an environment physically possible in which that difference manifests itself in a functionally salient way: it leads to a systematic difference in the production of functional side-effects that are tied to the effects that characterize $F$.

3) We can take those functional side-effects into account by conceiving functional sub-types (sub-concepts) of $F$. These sub-types all include the definition of $F$. They are distinct in taking into account differences in functional side-effects that the physical realizers of $F$, coming under different physical types, systematically produce in certain environments. For any functional type $F$ and any possible physical realizer type $P_{1}$ of $F$, it is in principle possible to conceive a functional sub-type $F_{1}$ of $F$ that has the same extension as $P_{1}$. This identity of extension applies to all nomologically possible worlds. Consequently, the concepts $F_{1}$ and $P_{1}$ are nomologically coextensive.

It is in dispute whether the configurations of physical tokens that come under a functional type $F$ are defined only by their composition or also by the effects that they have or can have. This dispute is immaterial to the present argument. If one includes a causal element in the definition of the configurations, then the first and the second step of this argument are even more straightforward. (Shapiro 2000, section 4, uses the possibility of a causal definition of 
the realizer configurations as an argument against multiple realization. He suggests that functional types are irrelevant. If one accepts Shapiro's argument, it shows that, in our terms, the functional sub-types, not the functional types, are of scientific interest).

The common multiple realization scheme is this one:

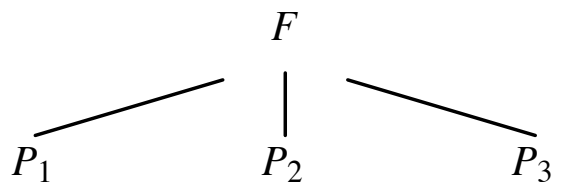

However, if $P_{1}, P_{2}$ and $P_{3}$ are different physical types of realizers of $F$, it is possible to introduce different functional sub-types of $F$, each sub-type of $F$ being coextensive with one realizer-type:

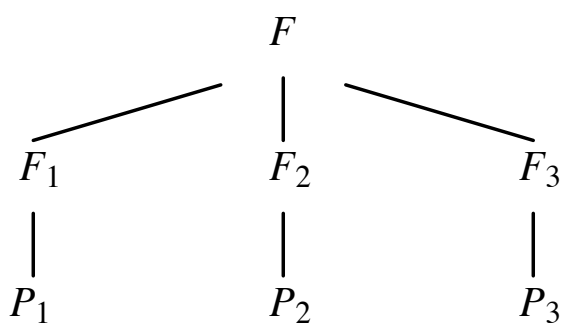

The crucial steps of this argument are the first and the second one, maintaining that differences in composition go together with causal differences that amount to functional differences providing for functional sub-types of $F$. These steps are based upon two claims:

a) A claim concerning the fundamental physical properties: The fundamental physical properties may be intrinsic or relational or both. We are not committed to a particular view in this paper. Assume that these properties are intrinsic. Nonetheless, if there are two different types of intrinsic fundamental properties, the difference between the tokens coming under these types somehow has to show up in some difference or other in the laws in which these types figure, including notably the causal laws. We are committed to that claim for our argument to go through. However, that claim alone does not tie our argument to a causal theory of properties (Shoemaker 1980) in contrast to a Humean theory of properties (cf. Lewis' thesis of Humean supervenience in Lewis 1986, IX-X). If the fundamental physical properties are relational instead of intrinsic, then the claim is straightforward that the difference between two fundamental physical property types shows up in the relations including notably the causal relations - in which the tokens coming under these types stand.

b) A claim concerning the transfer of this difference to a functionally relevant difference: Assume that there are two different types of fundamental physical realizers $P_{1}$ and $P_{2}$ of a given functional type $F$. The configurations of fundamental physical tokens coming under $P_{1}$ and the configurations of fundamental physical tokens coming under $P_{2}$ thus have some salient effects in common, namely those that characterize $F$. Nonetheless, if, as claim (a) establishes, there is a causal difference between the fundamental physical tokens of which these configurations are composed, there is a difference in the manner in which these configurations bring about the effects that characterize $F$. If there is such a difference, then there always is an environment possible in which that difference shows up in functional side- 
effects that are linked to the main functional effects characterizing $F$. Hence, that difference can be detected and expressed in the functional vocabulary.

For any fundamental physical effect, there is an environment conceivable in which that effect is amplified so that some sign of it shows up on a scale that is pertinent to the special sciences. The same goes for the configuration: the difference in the material of which the configurations of tokens coming under two different physical types of realizers of $F$ are composed can in principle be amplified in such a way that there is an environment conceivable in which these configurations react differently: they bring about in a systematic manner different functional side-effects due to the difference in the way in which they produce the effects that characterize $F$. Both these sorts of differences may in fact often be erased on the scale with which the functional theory is concerned. Nonetheless, it is always possible to conceive an environment in which these differences manifest themselves. For example, one may think of macroscopic quantum effects (such as, for instance, superconductivity, or tunnelling) that distinguish on a macroscopic scale between two different types of quantum physical realizers of a given functional type. Furthermore, chaos theory shows that slight microphysical differences can produce big macroscopic differences in a systematic way, etc.

An analogous consideration applies in the following case: the configurations of physical tokens coming under two different types of realizers of a given functional type $F$ are composed of fundamental physical tokens of the same type, but in ways that are distinct enough to account for there being two types of realizers (for example, one may think of different shapes of molecules that are composed of atoms of the same sort(s)). In this case again, there is an environment physically possible in which the difference in the composition of the fundamental physical tokens leads to a difference in functional side-effects: due to the difference in the manner in which these configurations bring about the effects that characterize $F$, they systematically produce certain functional side-effects.

In short, for every difference in composition that amounts to there being two types of realizers $P_{1}$ and $P_{2}$ of a functional type $F$, there are environments in which tokens of $P_{1}$ and $P_{2}$ bring about in a systematic and law-based way functional side-effects of different types. These differences enable us to conceive functional sub-types of $F$, as done in the third step of the above-mentioned argument. In order to be sound, this argument needs only to establish that it is in principle possible to introduce such functional sub-types, however widespread the multiple realization of a given functional type may be. It is not required that these functional sub-types are relevant to the classification scheme of the special science in question. It is sufficient that they can be conceived in its vocabulary. Their only purpose is to build up a nomological coextension with physical concepts.

This argument applies in both directions: it can be construed from below to above, arguing that different types of realizers, defined essentially by different manners of composition, lead to different types of functional side-effects that the tokens coming under these types can produce. But it can also be conceived from above: each functional type that is multiply realizable admits of a further specification in terms of functional sub-types until one gets to functional sub-types that are no longer multiply realizable; the characteristic causes and effects that define these sub-types demand a realization by physical tokens that exhibit a specific causal profile or that are arranged in a specific manner. 


\section{A biological illustration}

Let us consider an oversimplified example from genetics to illustrate this argument. The example is only intended as an illustration of the general scheme introduced in the preceding section. It is not supposed to be a case study in applying that scheme to get around the multiple realization argument against the reduction of classical genetics to molecular biology (see notably Kitcher 1984 for that argument). We do not have the space here to go into real biological cases or the literature on genetics and molecular biology (see Sachse 2007, chapters 5 to 7 ).

Let us come back to the causal relation between genotype and phenotype. Consider, as a somewhat imaginary and grossly oversimplified case, a gene whose characteristic phenotypic effect is to produce white blossoms at flowering time within certain types of plants. Suppose that the molecular realizers of this type of gene differ in one or more bases of the DNA so that there are at least two types of realizers. Imagine two individual plants, call them Jim and Joe, whose cells are molecularly identical except for the mentioned difference in bases of the DNA. Thus, they come under two distinct molecular realizer types of the gene in question. Nonetheless, assume that there is no difference between these two plants as far as the white colour of the blossoms is concerned that accounts for the functional definition of the gene.

However, to produce white blossoms, Jim's DNA segments interact differently with the intracellular components than Joe's DNA segments do. Under certain environmental conditions these differences manifest themselves in phenotypic differences. For instance, Jim's DNA segments may be more resistant to ultraviolet light and, thus, Jim's DNA segments may need no resources and time for reparation to produce white blossoms. In normal environments no phenotypic differences may appear because there is little ultraviolet light, and the selection pressure may be more on other factors than the resources and the time to produce white blossoms. Thus, in normal environments, Jim's DNA segments imply no selective advantages for the plant in comparison to Joe's DNA segments. However, there always is some demanding environment possible with high ultraviolet light and a selection pressure on the resources and time to produce white blossoms. Such a demanding environment favours Jim's DNA segments to produce white blossoms compared to Joe's. Jim's DNA segments are more resistant to ultraviolet light and produce white blossoms with less resources and more quickly. Joe's DNA segments are less resistant to ultraviolet light and, thus, need more resources for reparation and, therefore, more time to produce white blossoms. One can easily conceive situations in which these differences are pertinent to Jim's and Joe's fitness.

In brief and grossly oversimplifying, we can conceive two functional sub-types within the type "gene for white blossoms", namely "gene for an always quick production of white blossoms" $\left(F_{1}\right)$, as instantiated by Jim, and "gene for a sometimes slow production of white blossoms" $\left(F_{2}\right)$, as instantiated by Joe. These are, of course, only gross abbreviations of a detailed functional description that takes into account a quantitative analysis of the probabilities of fitness contributions. This means, it is possible to replace the mentioned abbreviations such as "gene for an always quick production of white blossoms" either with a specific value of fitness contribution, say $n$, or with a mathematical function that characterizes the probabilities of certain fitness contributions of the genes in question, say in $x \%$ the fitness contribution is $n$, in $\mathrm{y} \% n-0,1$, in $\mathrm{z} \% n-0,2$, etc. 
In general, selection is regarded as the main reason why functional, biological types admit of multiple realization (see notably Papineau 1993, chapter 2). Selection is supposed to be blind to composition. It is only concerned with functional effects that are relevant to the fitness of the organism in question. However, for any molecular difference that accounts for there being two types of realizers of a given functional, biological type $F$, there always is an environment physically possible in which that difference leads to phenotypic differences within the domain of the characteristic phenotypic effects of $F$. For any such phenotypic difference, there is an environment possible in which that difference amounts to a difference in fitness of the organisms in question, thus implying selective advantages or disadvantages (see Rosenberg 1994, 32). Hence, although selection provides for a good argument for the multiple realization of functional, biological types, invoking selection is no objection against the possibility to introduce functional sub-types that are coextensive with the realizer types. On the contrary, one can refer to selection in order to illustrate that possibility.

Functionalism in philosophy and the multiple realization argument were put forward in the late sixties by reference to artificial kinds in the first place (notably Putnam 1967 / 1975). Any machine is defined by its function in contrast to its composition. We select certain mechanisms because they produce certain desired effects, in whatever way they bring about these effects. But this classification is relative to our interests in having certain effects produced. The different types of realizers of a machine of a certain type differ in some sideeffects that they produce systematically in the manner in which they fulfil their function. For any machine, it is therefore in principle possible to introduce functional sub-types, corresponding to each realizer type. Such functional sub-types can thus be conceived in the case of artificial kinds as well as in the case of natural kinds.

\section{Intertheoretic reduction}

The argument presented and illustrated in the two preceding sections intends to be a proposal for intertheoretic reduction. It is based upon two crucial claims:

a) For any functional type $F$, it is always possible to introduce functional sub-types that are nomologically coextensive with the types of the physical realizers of $F$, for all possible physical realizer types of $F$. This claim has been argued for in the last but one section.

b) There are a small number of fundamental and universal physical theories - two in the current state of the art (quantum field theory and general relativity), one in the ideal case. We can construct in the last resort within these theories the descriptions of all the configurations of physical tokens that realize a given functional type $F$, for all $F$. Thus, the physical realizers of a given functional type do not come under different realizer theories. The issue is one of introducing different descriptions within a given fundamental theory.

In making that claim, as regards the current state of fundamental physics, we are committed to an interpretation of quantum theory according to which there are fundamental physical tokens that are not touched by quantum entanglement. These tokens then arrange themselves in configurations some of which are functional tokens (such as, for instance, biological tokens). In other words, we are committed to an interpretation of quantum theory that includes state reductions that dissolve superpositions and entanglement. However, this is a commitment that any position has to endorse that recognizes the existence of entities as they are described by the theories of the special sciences. 
The important point is that there is one theory - or a small number of theories - of fundamental physics within which it is in principle possible to construct the descriptions of all the configurations of physical tokens that realize a given functional type $F$, for all $F$. We are not committed to saying that there is a theory above fundamental physics and below the functional theory in question that can perform that task for a given $F$. For instance, assume that mental types are functional types whose tokens are identical with configurations of neurobiological tokens in humans. However, as the multiple realization argument points out, it may be the case that other living beings that do not have a brain nonetheless have tokens that come under the same mental types, these tokens being identical with configurations of other tokens than neurobiological ones. In that case, psychology cannot be reduced to neurobiology. But in any case, it can in principle be reduced to whatever may be the most specific theory that is able to cover all the realizers of the mental, functional types in question (e.g. molecular biology, or, finally, fundamental physics). In short, psychology is reducible to neurobiology if and only if all mental types are realized by configurations of neurobiological tokens; if not, one has to go further down in looking for a reducing theory.

We can set out this conception for theory reduction in the following way, limiting ourselves for the sake of simplicity to one fundamental physical theory $P$ and one functional type $F$ of some special science:

(1) Within an encompassing fundamental physical theory $P$, we construct the concepts $P_{1}$, $P_{2}, P_{3}$, etc. for the different configurations of physical tokens that all come under the same functional type (concept) $F$. $P$ is such that for any functional type $F$ that is realized in a nomologically possible world, concepts of the physical realizers of $F$ can be constructed within $P$. These constructed physical concepts $P_{1}, P_{2}, P_{3}$ are thus embedded in $P$.

(2) Within $F$, we construct more precise functional concepts in the form of functional subtypes (sub-concepts) $F_{1}, F_{2}, F_{3}$, etc. as explained in the two preceding sections. These concepts are nomologically coextensive with the concepts $P_{1}, P_{2}, P_{3}$, etc. Our proposal thus implies that not only the reducing theory, but also the theory that is to be reduced has to be prepared for reduction.

(3) We reduce $F$ to $P$ via $F_{1}, F_{2}, F_{3}$ and $P_{1}, P_{2}, P_{3}$. Starting from $P$, we construct $P_{1}, P_{2}, P_{3}$ within $P$, deduce $F_{1}, F_{2}, F_{3}$ from $P_{1}, P_{2}, P_{3}$ given the nomological coextension and gain $F$ by abstracting from the conceptualization of the functional side-effects that distinguish between $F_{1}, F_{2}, F_{3}$ :

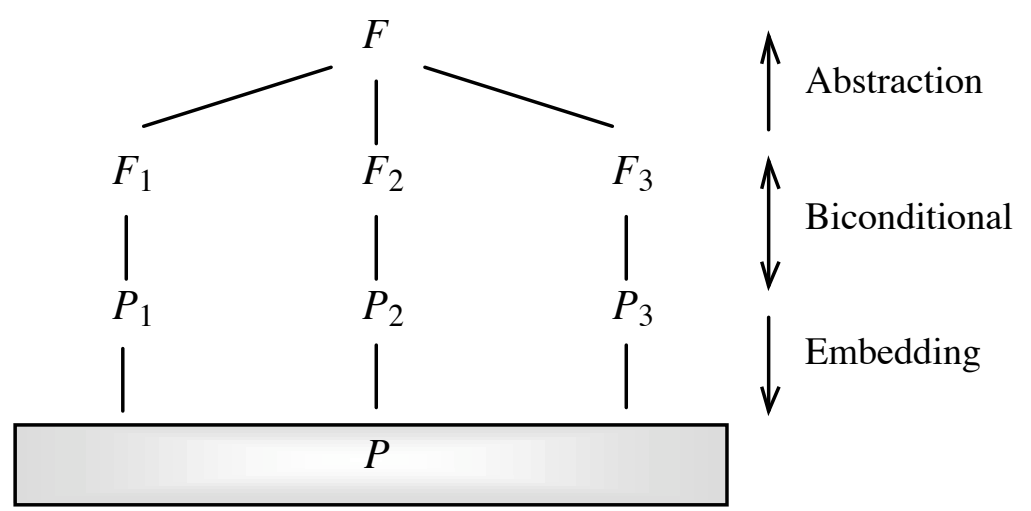

The content of the concepts in the $F$-domain and the $P$-domain remains in any case distinct content in the sense of primary intension, that is, in short, the inferential role of the concepts in question in a given theory or language (see e.g. Chalmers 1996, 52-71). If genetics is reducible to molecular biology, the content of the concepts "gene for white blossoms" and 
"segment X of DNA", for instance, nonetheless remains distinct. Token identity does not require synonymy of the concepts or descriptions that one and the same entity makes true; it requires only their systematic connectability.

As soon as we have obtained nomologically coextensive concepts of both theories, we have satisfied the main condition for theory reduction. The nomological coextension of the concepts $P_{1}, P_{2}, P_{3}$ with the concepts $F_{1}, F_{2}, F_{3}$ constitutes bridge principles that are the necessary and sufficient condition for theory reduction in the classical, logical empiricist conception set out by Nagel (1961, chapter 11). As already mentioned in section 2, bridge principles are in any case indispensable, because one has to link the concepts of the reduced theory with those of the reducing theory. That link is established in our proposal by constructing the concepts $F_{1}, F_{2}, F_{3}$, etc. that are coextensive with the concepts $P_{1}, P_{2}, P_{3}$, etc. in all nomologically possible worlds. (But there is no question here of discovering additional laws of nature in the form of bridge principles; the aim is a systematic correlation of different vocabularies that describe the same property tokens). If one were to abandon the search for bridge principles, reduction would no longer be conservative. It would then amount to replacing the theory targeted for reduction and its vocabulary with the reducing theory and its vocabulary. For instance, in renouncing bridge principles, the current stream known as "new wave reductionism" ends up in theory replacement instead of conservative reduction (see in particular Bickle 1998, 2003 for that stream and Endicott 1998 for a criticism that brings out the need for bridge principles).

Once we have developed such nomologically coextensive concepts, we can formulate the laws of $P$ insofar as they concern the realizers of $F$ by using the concepts $P_{1}, P_{2}, P_{3}$. Furthermore, we can formulate the laws or law-like generalizations in which $F$ figures by using $F_{1}, F_{2}, F_{3}$. Since $F_{1}, F_{2}, F_{3}$ make $F$ more precise, all the laws in which $F$ figures can be couched in the terms $F_{1}, F_{2}, F_{3}$. We can hence deduce the laws in which $F$ figures from the laws of $P$ via $P_{1}, P_{2}, P_{3}$ and $F_{1}, F_{2}, F_{3}$. Due to multiple realization, there is no physical concept of $P$ that is coextensive with the functional concept $F$. But through the introduction of functional sub-types $F_{1}, F_{2}, F_{3}$, we can nevertheless carry out a fully-fledged theory reduction. This is, of course, merely an ideal scheme intended only to establish the principled possibility of theory reduction despite multiple realization.

There is an obvious philosophical limitation of our proposal: it is unable to cope with homogeneous functional patterns across different possible worlds outside the sphere of nomologically possible worlds, if there are such patterns. Consider the most extreme case one might envisage: one mental functional type being realized both by the physical matter of our world (configurations of neurobiological tokens) and disembodied souls in a remote possible world. In that case, there is no possibility of constructing within a given fundamental and universal physical theory the descriptions of all the configurations that realize the functional type in question. There is thus no possibility to reduce the special science theory of $F$ via the introduction of functional sub-types $F_{1}, F_{2}, F_{3}$ to one physical theory $P$ via the construction of nomologically coextensive concepts $P_{1}, P_{2}, P_{3}$ in $P$. However, one may with good reason doubt whether in that case there is a homogeneous pattern of characteristic causes and effects that comes under one and the same functional type (cf. Block's Disney Principle in Block 1997, 120-124, as well as Hooker 2004, 469-475). Be that as it may, at most we would have to concede that our strategy for intertheoretic reduction is limited to all nomologically possible worlds. 
Our proposal develops Kim's conception of functional reduction commented upon in section 2 into a proposal for a complete and conservative theory reduction. It thereby links Kim's conception with Nagel's logical empiricist conception (see also Marras 2005, sections 2 and 3, on the link between Kim and Nagel). Our main step beyond Kim is this one: instead of having only one-way conditionals of the form "If something comes under the physical type $P$, then it comes under the functional type $F$ " at our disposal, our proposal provides for biconditionals linking functional with physical concepts. It thus satisfies the central condition for a fully-fledged - and conservative - theory reduction. Nonetheless, our proposal includes the virtues of explanation that Kim's conception contains, namely the causal explanation of why there are entities in the world that come under functional types (see step 3 at the beginning of section 2).

Our proposal does not rely on introducing the notion of structure-specific realizers and thereby carry out what Kim calls a local or structure-specific reduction (for instance, the concept of pain reduces in one structure, say humans, to one physical concept - e.g. "firing of C-fibres" -, the concept of pain reduces in another structure, say octopuses, to another physical concept, etc.) (see Kim 1998, 93-95; 2005, 25). The functional sub-types are not conceived in terms of any specific structures, and they do not presuppose a uniform realization of the functional type in question within a given structure. They do not split up the homogeneous functional type into structure-relative functions ( $F$ in structure $S_{1}, F$ in $S_{2}, F$ in $S_{3}$, etc.), thereby losing the unity of the functional type - that is, the salient similarities among the configurations of physical tokens of different structure (composition) in virtue of which they come under one functional concept $F$. Concepts like " $F$ in $S_{1}$ ", " $F$ in $S_{2}$ ", etc. are functional-cum-physical concepts instead of purely functional concepts. They relativize $F$ to specific structures such that $F$ means something different in each case. Consequently, these concepts cannot express a significant common meaning of the concept $F$. There is nothing that is of scientific importance left for $F$ itself.

By contrast, the functional sub-types introduced here are completely functional concepts. They remain entirely within the domain of the functional vocabulary of the special science in question. The functional sub-types are distinct only by conceptualizing side-effects that are tied to the causal role that defines the functional type: they are heterogeneous as regards these functional side-effects, but homogeneous insofar as the functional effects are concerned that define the functional type. $F$ always has the same substantial meaning in $F_{1}, F_{2}, F_{3}$, etc. - a meaning that is only further specified by taking functional side-effects into account. Therefore, $F_{1}, F_{2}, F_{3}$, etc., clearly express for the scientist of the special science in question what the referents of these concepts functionally have in common and what their functional differences are (which may be of interest for the special science in question).

One can easily deduce from $F_{1}, F_{2}, F_{3}$, etc. the functional type $F$ by simply abstracting from the conceptualization of the side-effects. Therefore, our proposal preserves the unity of the functional type. To come back to the illustrative example of a gene for white blossoms, the concept $F_{1}$ "gene for an always quick production of white blossoms" and the concept $F_{2}$ "gene for a sometimes slow production of white blossoms" both include the causally homogeneous functional type ("gene for white blossoms") and the laws relating to that type. That type ("gene for white blossoms") has the same substantial meaning in all these subtypes. 
Since the functional sub-types introduce a more fine-grained functional classification than the respective functional types, these latter are determinable concepts that can be further determined by conceiving functional sub-types within the vocabulary of the functional theory in question. The functional type "gene for white blossoms" is a determinable concept with respect to the more determinate concepts "gene for an always quick production of white blossoms" and "gene for a sometimes slow production of white blossoms". These latter stand to the functional type in the species-genus relation. There is no obvious limit as to what extent one can pursue the construction of ever more determinate concepts within a given vocabulary. As regards the functional sub-types introduced here, they are as determinate as is necessary to get to functional concepts that are nomologically coextensive with physical concepts describing the physical composition of the realizer types (or chemical, or molecular biological, or neurobiological concepts for that matter).

We do not regard concepts such as the functional type $F$ as determinable and the concepts $P_{1}, P_{2}, P_{3}$, etc. as their determinates (as to such proposals, see notably Yablo 1992, 250-271; Clapp 2001, 125-132; Hooker 2004, 467). The reason is that the principles of type distinction are different: the functional types are defined by their characteristic causes and effects. The types of realizers, by contrast, are essentially differentiated by their composition. There is no semantic way of meaning analysis from the $P$-concepts to the $F$-concepts (and vice versa). In short, we do regard $F$ as a determinable concept, but its determinates are further functional concepts. This then enables us to identify the extension of the functional determinates (the sub-types) with the extension of the types of realizers in a systematic way, that relation being not the determinable-determinate relation, but nomological coextension.

The reductionism proposed here is conservative. Some configurations of physical tokens, being functional tokens, make true a functional description in the same way as they make true a physical description. It is not the case that these entities make true a physical description in the first place and a functional description in a somewhat secondary, derived way. There is only one relation of truth-making. The entities in the world make true all descriptions in the same way, and the descriptions can be connected as explained in this section.

The functional theories of the special sciences are part and parcel of a coherent system of our knowledge. They are indispensable in bringing to the point salient similarities among configurations of fundamental physical tokens of different physical types. These configurations make up a certain functional pattern in virtue of these similarities. Of course, any such pattern supervenes on the distribution of the fundamental physical tokens in spacetime. But this does not mean that the vocabulary of a fundamental physical theory is apt to express these patterns in a concise manner. As mentioned above, there is no physical concept in $P$ that is coextensive with the functional type $F$. The physical vocabulary is made for the description of the heterogeneous composition of these configurations. It does not focus on the configurations as such and - notably - not on the salient functional effects that they have in common. Epistemological reductionism is intended to establish a connection between the special sciences and physics - as done here by constructing the nomologically coextensive concepts $F_{1}, F_{2}, F_{3}$, etc. and $P_{1}, P_{2}, P_{3}$, etc. - instead of eliminating the theories of the special sciences from a coherent system of our knowledge.

We do not claim that, if we had a complete description of the distribution of the fundamental physical tokens in the vocabulary of a comprehensive fundamental physical theory at our disposal, an a priori deduction of any true statement of a theory of the special 
sciences would in principle be possible, given analytic definitions of all the concepts of the special sciences. Although the truth-value of all the descriptions of the special sciences supervenes on a true and complete description of the distribution of the fundamental physical tokens, we conceive intertheoretic reduction as an a posteriori, empirical affair (cf. Laurence \& Margolis 2003 and Marras 2005 contra Chalmers \& Jackson 2001): given the theories $P$ and $F$, the concepts $P_{1}, P_{2}, P_{3}$, etc. and $F_{1}, F_{2}, F_{3}$, etc. have to be created within $P$ and $F$. How the creation of such nomologically coextensive concepts is to be carried out is an empirical affair, based on empirical knowledge of how the descriptions couched in the $F$ vocabulary are made true by entities that are identical with configurations of fundamental physical tokens.

What we have offered is a strategy showing the principled possibility of theory reduction. It is intended to be a solution to a philosophical problem. The functional sub-types introduced here are conceived for the purpose of showing how the descriptions of the special sciences including their law-like generalizations - can be true, given that they are made true by entities that are identical with configurations of fundamental physical tokens. The resulting position is a conservative reductionism, opening up a middle way between anti-reductionism and eliminativism - a middle way offering a coherent position that does not leave anything mysterious in our understanding of the world without eliminating anything.

Acknowledgements: We are very grateful to the Editor and three anonymous referees for a number of helpful comments. The work of Christian Sachse on this paper has been supported by the Swiss National Science Foundation (SNF), grant nr. 100011-105218/1.

\section{References}

BECHTEL, W. \& MUNDALE, J. (1999) Multiple realizability revisited: linking cognitive and neural states, Philosophy of Science, 66, pp. 175-207.

BICKLE, J. (1998) Psychoneural Reduction: the New Wave (Cambridge (Massachusetts), MIT Press).

BICKLE, J. (2003) Philosophy and Neuroscience. A Ruthlessly Reductive Account (Dordrecht, Kluwer).

BLOCK, N. (1997) Anti-reductionism slaps back, in: J. E. TOMBERLIN (Ed.) Philosophical Perspectives 11: Mind, Causation, and World (Oxford, Basil Blackwell). Pp. 107-132.

CHALMERS, D. J. (1996) The Conscious Mind. In Search of a Fundamental Theory (New York, Oxford University Press).

CHALMERS, D. J. \& JACKSON, F. (2001) Conceptual analysis and reductive explanation, Philosophical Review, 110, pp. 315-360.

CLAPP, L. (2001) Disjunctive properties: multiple realizations, Journal of Philosophy, 98, pp. 111-136.

ENDICOTT, R. P. (1998) Collapse of the new wave, Journal of Philosophy, 95, pp. 53-72.

FODOR, J. A. (1974) Special sciences (or: The disunity of science as a working hypothesis), Synthese, 28, pp. 97-115. Reprinted in N. BLOCK (Ed.) (1980) Readings in the Philosophy of Psychology. Volume 1 (Cambridge (Massachusetts), Harvard University Press). Pp. 120-133.

HEIL, J. (2003) From an Ontological Point of View (Oxford, Oxford University Press).

HOOKER, C. A. (2004) Asymptotics, reduction and emergence, British Journal for the Philosophy of Science, 55, pp. 435-479.

HÜTTEMANN, A. (2003) What's Wrong with Microphysicalism? (London, Routledge).

HULL, D. (1974) Philosophy of Biological Science (Englewood Cliffs, Prentice-Hall)

KIM, J. (1998) Mind in a Physical World. An Essay on the Mind-Body Problem and Mental Causation (Cambridge (Massachusetts), MIT Press).

KIM, J. (1999) Making sense of emergence, Philosophical Studies, 95, pp. 3-36.

KIM, J. (2005) Physicalism, or Something Near Enough (Princeton, Princeton University Press). 
KITCHER, P. (1984) 1953 and all that. A tale of two sciences, Philosophical Review, 93, pp. 335-373. Reprinted in P. KITCHER (2003) In Mendel's Mirror. Philosophical Reflections on Biology (Oxford, Oxford University Press). Pp. 3-30.

LAURENCE, S. \& MARGOLIS, E. (2003) Concepts and conceptual analysis, Philosophy and Phenomenological Research, 67, pp. 253-282.

LEWIS, D. (1970) How to define theoretical terms, Journal of Philosophy, 67, pp. 427-446. Reprinted in D. LEWIS (1983) Philosophical Papers. Volume 1 (Oxford, Oxford University Press). Pp. 78-95.

LEWIS, D. (1986) Philosophical Papers. Volume 2 (Oxford, Oxford University Press).

MARRAS, A. (2005) Consciousness and reduction, British Journal for the Philosophy of Science, 56, pp. $335-361$.

McCAULEY, R. (2007) Reduction: models of cross-scientific relations and their implications for the psychology-neuroscience interface, forthcoming in: P. THAGARD (Ed.): Handbook of the Philosophy of Psychology and Cognitive Science (Amsterdam, Elsevier). (In press).

NAGEL, E. (1961) The Structure of Science. Problems in the Logic of Scientific Explanation (London Routledge).

PAPINEAU, D. (1993) Philosophical Naturalism (Oxford, Basil Blackwell).

PUTNAM, H. (1975) The nature of mental states, in: H. PUTNAM: Mind, Language and Reality. Philosophical Papers Volume 2 (Cambridge, Cambridge University Press). Pp. 429-440. First published as "Psychological predicates" in W. H. CAPITAN and D. D. MERRILL (Eds.) (1967) Art, Mind and Religion (Pittsburgh, University of Pittsburgh Press).

ROSENBERG, A. (1994) Instrumental Biology or the Disunity of Science (Chicago, University of Chicago Press).

SACHSE, C. (2007) Reductionism in the philosophy of science (Frankfurt (Main), Ontos).

SHAPIRO, L. (2000) Multiple realizations, Journal of Philosophy, 97, pp. 635-654.

SHOEMAKER, S. (1980) Causality and properties, in: P. VAN INWAGEN (Ed.) Time and Cause (Dordrecht, Reidel). Pp. 109-135. Reprinted in S. SHOEMAKER (1984) Identity, Cause, and Mind. Philosophical Essays (Cambridge, Cambridge University Press). Pp. 206-233.

SHOEMAKER, S. (1981) Some varieties of functionalism, Philosophical Topics, 12, pp. 83-118. Reprinted in S. SHOEMAKER (1984) Identity, Cause, and Mind. Philosophical Essays (Cambridge, Cambridge University Press). Pp. 261-286.

YABLO, S. (1992) Mental causation, Philosophical Review, 101, pp. 245-280. 